\title{
Gravidez na adolescência: a influência dos antecedentes familiares e sociais na cidade de Anápolis - GO
}

\author{
Teenage pregnancy: the influence of family and social background in the city of Anápolis - GO \\ Embarazo en la adolescencia: la influencia de familia y trasfondo social en la ciudad de Anápolis -
} GO

\section{Resumo}

Introdução: A gravidez na adolescência (GA) é um importante assunto de saúde pública, por sua alta prevalência e implicações para a gestante e o recém-nascido. Objetivo: Descrever os dados sociais e antecedentes familiares que atuam como predisponentes para GA, em puérperas adolescentes atendidas em um hospital público do município de Anápolis, Goiás, Brasil. Metodologia: Estudo de caráter transversal, epidemiológico e abordagem quantitativa. Foi aplicado um questionário em adolescentes puérperas na faixa etária de 10 a 19 anos internadas após parto. Resultados: Observou-se uma predominância de antecedentes de GA da mãe da puérpera ficando clara a influência dos pais na tomada de decisão dos filhos. A presença de apoio e bom convívio familiar, foram prevalentes contradizendo a literatura existente. Notou-se grande número de gestações não planejadas, indicando baixo conhecimento para prevenção de gestação. Quanto aos aspectos sociais, a minoria havia passado por violência familiar, também contrariando a literatura existente que mostra números mais elevados. Em geral as meninas tiveram educação sexual na escola, o que demonstra uma baixa efetividade das aulas em modificar condutas. Um elevado número de entrevistadas possuía acesso a uma UBS próxima, o que demonstra falha na orientação dos adolescentes quanto a vida sexual. Por fim, as adolescentes, em geral, não fizeram uso de drogas ilícitas ou ilícitas. Conclusão: O presente estudo evidencia que não é necessário um ambiente violento e insalubre para ocorrência de GA, porém os familiares possuem grande influencia nas condutas do adolescente, sendo importante melhor articulação da educação em saúde nas escolas e UBS.

Palavras-chave: Adolescente; Saúde da mulher; Gravidez na adolescência.

\footnotetext{
Abstract

Introduction: Teenage pregnancy (GA) is an important public health issue, due to its high prevalence and implications for pregnant women and newborns. Objectives: Describe the social data and family history that act as predisposing factors for GA in adolescent puerperal women whom attended at a public hospital in the city of Anápolis, Goiás, Brazil. Methodology: Cross-sectional, epidemiological study and quantitative approach. A questionnaire was applied to puerperal adolescents aged 10 to 19 years hospitalized after childbirth. Results: There was a predominance of GA
} 
history of the mother of the puerperal teen, demonstrating the influence of the parents in the decision making of their children. The presence of support and good family routine were prevalent, contradicting the existing literature. A large number of unplanned pregnancies was noted, indicating low knowledge for pregnancy prevention. As for social aspects, the minority had experienced family violence, also contrary to the existing literature that shows higher numbers. In general, girls had sex education at school, which demonstrates low effectiveness of classes in modifying behaviors. A high number of interviewees had access to a nearby primary health unit, which demonstrates a failure in the orientation of adolescents regarding their sexual life. Finally, adolescents, in general, did not use illicit or illicit drugs. Conclusion: The present study shows that a violent and unhealthy environment is not mandatory for the occurrence of GA, but family members have great influence on the adolescent's behavior, with better articulation of health education in schools and primary health units being important.

Keywords: Adolescent; Women's health; Pregnancy in adolescence.

\section{Resumen}

Introducción: El embarazo adolescente (EG) es un problema de salud pública importante, debido a su alta prevalencia e implicaciones para la gestante y el recién nacido. Objetivo: Describir los datos sociales y antecedentes familiares que actúan como factores predisponentes de EG en puérperas adolescentes atendidas en un hospital público de la ciudad de Anápolis, Goiás, Brasil. Metodología: Estudio transversal, epidemiológico y abordaje cuantitativa. Se aplicó un cuestionario a adolescentes puerperales de 10 a 19 años hospitalizadas después del parto. Resultados: Predominó el antecedente de EG de la madre de la mujer puerperal, evidenciando la influencia de los padres en la toma de decisiones de sus hijos. Predominó la presencia de apoyo y una buena convivencia familiar, contradiciendo la literatura existente. Se observó un gran número de embarazos no planeados, lo que indica un bajo conocimiento para la prevención del embarazo. En cuanto a los aspectos sociales, la minoría había experimentado violencia familiar, también contrariamente a la literatura existente que muestra cifras más altas. En general, las niñas recibieron educación sexual en la escuela, lo que demuestra la baja efectividad de las clases para modificar comportamientos. Un elevado número de entrevistadas tuvo acceso a una unidad de salud cercana, lo que demuestra una falla en la orientación de los adolescentes con respecto a su vida sexual. Finalmente, los adolescentes, en general, no consumían drogas ilícitas o ilícitas. Conclusión: El presente estudio muestra que no es necesario tener un ambiente violento e insalubre para la ocurrencia de $\mathrm{AG}$, pero los familiares tienen una gran influencia en el comportamiento del adolescente, y es importante articular mejor la educación para la salud en las escuelas y unidades de salud.

Palabras clave: Adolescente; Salud de la mujer; Embarazo en adolescencia.

\section{Introdução}

A gravidez na adolescência é considerada um importante assunto de saúde pública, em decorrência da alta prevalência desse fenômeno ao redor do mundo e suas implicações para com a gestante e o recém-nascido (Cunnington, 2001; Goodyear, Newcomb, \& Locke, 2002; Lawlor, \& Shaw, 2004). Sendo caracterizada como aquela que ocorre em indivíduos entre 10 e 20 anos incompletos, a gestação na adolescência é responsável por cerca de $18 \%$ dos nascidos vivos mundialmente, e cerca de $23 \%$ no Brasil (Bouzas, Cader, \& Leao, 2014).

Dada a heterogeneidade das populações de cada país, diversos estudos têm sido feitos para observar e relatar como esse fenômeno se comporta em cada região. Enquanto Bennet, Skatrud, Guild, Loda e Klerman (1997) relataram uma maior ocorrência de gravidez na adolescência em zonas rurais, quando estas são comparadas com zonas urbanas, Henshaw e Feivelson (2000) relataram que os maiores índices recaem sobre a parcela negra da população de diversas regiões dos Estados Unidos da América (EUA). Ambos os autores argumentam sobre a importância de determinantes socioeconômicos sobre a maior incidência desse fenômeno nessas populações, uma vez que ambas as populações possuem menor acesso à educação e menores níveis de remuneração.

Segundo o Ministério da Saúde (2021), aumentou-se a participação da gravidez na adolescência nos índices de fecundidade, enquanto houve diminuição proporcional da participação das demais faixas etárias. Além disso, durante os anos de 2015 a 2019, observou-se, por ano, uma média de 481 mil gestações em adolescentes de 10 a 10 anos, sendo que, em torno de 13 mil ocorreram no estado de Goiás. Diversos estudos realizados no Brasil também destacaram a alta e crescente taxa de gestações na adolescência e demonstraram correlações desse fenômeno com aspectos familiares e sociais dessa população. De fato, pesquisas realizadas na cidade do Rio de Janeiro mostraram que existe uma correlação positiva entre gravidez na adolescência e piores índices de qualidade de vida, com maior prevalência de abortos, consumo de cigarros e drogas ilícitas na 
gestação, bem como maior incidência de desemprego e desenvolvimento de famílias monoparentais (Gama, Szwarcwald, \& Leal, 2002; Sabroza, Leal, Gama, \& Costa, 2004).

É importante salientar que aspectos familiares são importantes no desenvolvimento desse cenário de crescente taxa de fecundidade em adolescentes. De fato, existem evidências que apontam para uma maior falta de acesso à proteção social e familiar como um fator preponderante para a gestação na adolescência (Departamento Científico de Adolescência, 2018). Além disso, a inexistência de um núcleo familiar consolidado socioeconomicamente pode acarretar dificuldades na criação e sustento do recém-nascido, o que, segundo alguns autores, pode favorecer no desenvolvimento de um ciclo de exclusão social por garantir a manutenção dos baixos níveis socioeconômicos da puérpera (Melhado, Sant'Anna, Passarelli, Coates, 2009; Santos, Cremonese, Wilhelm, Castiglioni, \& Ressel, 2014). Ademais, os autores argumentam que parte importante para a criação desse ciclo é o abandono escolar da adolescente, que ocorre em cerca de 85,7\% até o terceiro trimestre de gravidez, gerando um comprometimento não apenas da continuidade da educação formal, como também uma menor qualificação profissional e deficiência na inserção no mercado de trabalho.

Por fim, para além dos aspectos familiares e socioeconômicos envolvidos na gestação na adolescência, existem sérios riscos para a vida da puérpera e do recém-nascido. De fato, segundo (Almeida et al., 2020), existe uma importante correlação positiva entre partos prematuros espontâneos e gravidez na adolescência, havendo $24 \%$ mais chances de ocorrer esse fenômeno em adolescentes precoces, quando compara-se com adolescentes tardias e adultas jovens. Estes dados vão de acordo com evidências apontadas por (Sadovsky et al., 2018), onde análises de quatro grandes coortes brasileiras também demonstraram que a idade precoce de gestação é um importante fator associado à prematuridade.

Diante disso, fica claro a importância do desenvolvimento de novos estudos que busquem abarcar a complexidade de fatores familiares, sociais, culturais e econômicos que levam ao aumento da taxa de fecundidade em adolescentes em diferentes regiões, com o intuito de guiar a construção de políticas públicas de saúde e educação para essa população.

Nesse sentido, o presente artigo tem como objetivo a descrição de dados sociais e antecedentes familiares que possam atuar como predisponentes para gestação na adolescência, como ocorrência de gestação precoce da mãe, apoio familiar, planejamento de gestação, acesso à educação sexual, acesso a unidades de saúde, uso de drogas lícitas e ilícitas, e religião, em puérperas adolescentes atendidas em um hospital público do município de Anápolis, no Estado de Goiás, Brasil.

\section{Metodologia}

O presente estudo tem caráter transversal, epidemiológico, de abordagem qualitativa, buscando identificar dados sociais e antecedentes familiares de adolescentes puérperas atendidas em uma unidade de referência do Sistema Único de Saúde (SUS) no município de Anápolis, no Estado de Goiás, Brasil (Estrela, 2018). O estudo foi realizado na Santa Casa de Anápolis (SCA), tida como unidade de referência para gestantes de alto risco, incluindo gestantes adolescentes. Os dados foram coletados durante o período entre fevereiro de 2020 e setembro de 2020, excetuando-se os meses a partir de meados de março a junho, em decorrência das impossibilidades advindas da pandemia do Severe Acute Respiratory Syndrome Coronavirus 2 (SARS-CoV-2).

A população definida no presente estudo foi a de adolescentes gestantes na faixa etária de 10 a 19 anos que tenham passado por regime de internação na SCA após parto nesta instituição. Para fins de construção do cálculo amostral do presente artigo, levou-se em consideração que $90 \%$ da amostra tem gravidez não planejada, utilizando um nível de significância de dois desvios padrão e um erro aceitável para o cálculo de 5\%. Para tanto utilizou-se a fórmula para o cálculo de amostra finita de (Levin, 1987).

A pesquisa foi realizada por meio de coleta de dados junto às adolescentes puérperas de 10 a 19 anos de idade internadas na SCA, as quais foram abordadas de modo aleatório durante a internação no local no período de coleta de dados, 
compreendido entre fevereiro/2020 e setembro/2020. A coleta de dados se baseou em perguntas direcionadas contidas no formulário específico "Fatores que influenciam na ocorrência de gravidez na adolescência". Este formulário foi elaborado levando em consideração os diversos elementos sociais e familiares que, segundo a literatura, predispõem a gestação na adolescência. A entrevista durou cerca de 10 minutos, sendo as perguntas realizadas de forma sigilosa e isolada entre as participantes e o interrogador.

Os critérios de inclusão para participar do estudo foram: adolescentes, internadas no pós-parto no período de coleta de dados, que aceitaram participar da pesquisa. Os critérios de exclusão para participar do estudo foram: adolescentes internadas que recusaram a participação na pesquisa ou aquelas menores de 18 anos desacompanhadas de seus pais ou responsáveis.

Para a organização dos dados foi utilizado o programa Microsoft® Excel 2013 para tabulação dos dados e a análise estatística. Os dados foram analisados no software SPSS para Windows, versão 21.0. Neste foi caracterizada a amostra através de médias, frequência simples e percentual.

A pesquisa foi submetida ao Comitê de Ética do Centro Universitário de Anápolis (UniEVANGÉLICA) conforme resolução 466/2012, já aprovado com número de parecer 3.364.153/2020.

\section{Resultados}

O questionário foi repassado a 54 pacientes adolescentes puérperas internadas na Santa Casa de Anápolis, no período de fevereiro a outubro de 2020, excetuando-se os meses a partir de meados de março a junho devido as impossibilidades impostas pela pandemia do SARS-CoV-2. Essas pacientes fazem uso do sistema único de saúde, sendo 17 delas na faixa etária dos 14 aos 16 anos e as outras 37 entre 17 e 19 anos de idade. De acordo com os dados obtidos, observa-se um perfil de antecedentes familiares das adolescentes puérperas marcadamente denotado pela presença de mães que tiveram o primeiro filho antes dos 20 anos $(81,5 \%, 44 / 54)$.

Tabela 1 - Antecedentes familiares das 54 mães adolescentes entrevistadas em 2020 na Santa Casa de Anápolis (GO).

\begin{tabular}{l|c|c}
\hline \multicolumn{1}{c|}{ Antecedentes familiares } & & $\mathrm{n}(\%)$ \\
\hline \multirow{2}{*}{ Mãe da adolescente teve filho antes dos 20 anos } & Sim & $44(81,5)$ \\
\cline { 2 - 3 } & Não & $9(16,6)$ \\
\cline { 2 - 3 } Presença de apoio familiar & Ignorado & $1(1,9)$ \\
\hline \multirow{2}{*}{ Presença de apoio do pai da criança } & Sim & $50(92,6)$ \\
\cline { 2 - 3 } & Não & $4(7,4)$ \\
\hline \multirow{2}{*}{ Presença de bom convívio familiar } & Sim & $45(83,3)$ \\
\hline \multirow{2}{*}{ Gestação planejada } & Não & $9(16,7)$ \\
\cline { 2 - 3 } & Sim & $49(90,7)$ \\
\cline { 2 - 3 } & Não & $5(9,3)$ \\
\cline { 2 - 3 } & Sim & $12(22,2)$ \\
\hline
\end{tabular}

Fonte: Dados da Pesquisa (2020).

Além disso, o trabalho contempla o reconhecimento dos aspectos sociais, o qual permitiu uma sistematização da violência familiar, uma vez que dentre as adolescentes que sofreram com violência, 15 adolescentes (27,8\%), 33,3\% (05/15) sofreram violência física, 33,3\% (05/15) violência psicológica, 6,7\% (01/15) violência sexual e 26,7\% (04/15) sofreram mais de um desses tipos de violências. Quando questionadas a respeito de quem foi o agressor, 66,7\% (10/15) responderam, sendo 
que dessas 30\% (03/10) respondem que a violência partiu de familiares, $20 \%(02 / 10)$ do parceiro e outros $20 \%(02 / 10)$ de outros agressores (Tabela 2).

Previamente à gestação $33,3 \%(18 / 54)$ das pesquisadas faziam uso de bebida alcoólica, dessas 27,8\% (05/18) continuou com a ingestão durante a gestação. Cenário diferente daquele encontrado para as adolescentes que faziam uso de cigarro, 7,4\% (04/54), já que 75\% (03/04) delas continuaram fumando durante a gestação e uma adolescente que não fazia uso de cigarro antes da gestação o fez durante ela. Igualmente aconteceu com o uso de drogas ilícitas, uma vez que a única adolescente $1,9 \%$ (01/54) a atestar o uso de droga ilícita anterior a gestação, continuou seu uso durante a gestação (Tabela 2). Por fim, das $85,2 \%$ (46/54) de gestantes com religião estabelecida, 74\% (34/46) delas se consideram praticantes de sua religião.

Tabela 2 - Aspectos sociais das 54 mães adolescentes entrevistadas em 2020 na Santa Casa de Anápolis (GO).

\begin{tabular}{|c|c|c|}
\hline Aspectos Sociais & & $\mathrm{n}(\%)$ \\
\hline \multirow{3}{*}{ Sofreu ou presenciou violência familiar } & Sim & $15(27,8)$ \\
\hline & Não & $38(70,4)$ \\
\hline & Ignorado & $1(1,9)$ \\
\hline \multirow{4}{*}{ Violência praticada por } & Parceiro & $2(3,7)$ \\
\hline & Familiar & $3(5,6)$ \\
\hline & Outro & $2(3,7)$ \\
\hline & Ignorado & $43(79,6)$ \\
\hline \multirow{3}{*}{ Já teve educação sexual na escola } & Sim & $37(68,5)$ \\
\hline & Não & $11(20,4)$ \\
\hline & Não se lembra & $6(11,1)$ \\
\hline \multirow{4}{*}{ Tempo para chegar à Unidade de Saúde } & $<30 \min$ & $41(75,9)$ \\
\hline & $30-60 \min$ & $8(14,8)$ \\
\hline & $>60 \mathrm{~min}$ & $3(5,6)$ \\
\hline & Ignorado & $2(3,7)$ \\
\hline \multirow{3}{*}{ Usou bebida alcoólica durante a gestação } & Sim & $5(9,3)$ \\
\hline & Não & $48(88,9)$ \\
\hline & Ignorado & $1(1,9)$ \\
\hline \multirow{2}{*}{ Fumou durante a gestação } & Sim & $4(7,4)$ \\
\hline & Não & $50(92,6)$ \\
\hline \multirow{2}{*}{ Fez uso de droga ilícita durante a gestação } & Sim & $1(1,9)$ \\
\hline & Não & $53(98,1)$ \\
\hline \multirow{2}{*}{ Possui religião } & Sim & $46(85,2)$ \\
\hline & Não & $8(14,8)$ \\
\hline
\end{tabular}

Fonte: Dados da Pesquisa (2020).

\section{Discussão}

Os antecedentes familiares encontrados nas participantes identificam um perfil de presença de gestação na adolescência anterior na família, existência de apoio familiar e do pai da criança, bom convívio em casa e uma gravidez não planejada. Sobre o apoio recebido por familiares e parceiro, o presente estudo ratifica a presença de uma relação de apoio e companheirismo entre as puérperas e seus familiares e parceiro. Tais dados divergem-se de resultados previamente obtidos por outros grupos de pesquisa, uma vez que esses estudos abordam a presença de uma boa relação familiar e conjugal como um 
fator determinante na prevenção da gravidez na adolescência, pelo fato de estas adolescentes possuírem menor chance de ter hábitos sexuais precoces e inadvertidos por receberem maior orientação, apoio e acompanhamento adequados (Alrahmani et al., 2016; Ribeiro, 2011). Contudo, os resultados obtidos no presente estudo podem apresentar vieses, posto que muitas entrevistadas podem ter avaliado apenas o momento atual do pós-parto, em que há um costume de maior apoio familiar e conjugal.

A influência dos hábitos familiares também pode ter interferido na maior incidência de gestação na adolescência, visto que $81,5 \%$ das mães das adolescentes também tiveram seus partos entre 10-19 anos. Segundo Savegnago e Arpini (2016), os pais atuam como exemplo e educadores para seus filhos, o que leva à um certo grau de influência sobre as decisões tomadas de seus filhos, em grande parte no âmbito da sexualidade.

Elevadas taxas de gestações não planejadas foram encontradas, o que pode indicar um conhecimento inadequado do uso dos métodos contraceptivos associado a alta incidência de gestação não planejada. Nesse sentido, alguns estudos evidenciam a carência de educação sexual como um fator determinante na gestação da adolescência, posto que muitas não tem acesso às estratégias do Planejamento Familiar da rede de saúde pública e privada e, as que têm, não as cumpre de forma adequada, por falta de apoio familiar e conjugal ou pela ausência de informação sobre os tipos e modos de utilização dos métodos contraceptivos (Aragão \& Cavalcanti, 2019; Santos, Silva, Queiroz, Jorge \& Brilhante, 2018).

Ademais, dentro dos aspectos sociais nota-se um perfil de adolescentes que não declararam contato com violência familiar, que já tiveram educação sexual na escola, com acesso a uma unidade de saúde próxima, fazem pouco uso de bebida alcoólica, cigarro ou droga ilícita e que possui uma religião.

Quanto à violência familiar, existe uma importante correlação da sua presença com prognóstico ruim da gestação, além de afetar todo o contexto familiar (Silva, 2019). O presente estudo apontou para um percentual de $27,8 \%$ das entrevistadas que afirmaram ter presenciado ou sofrido algum tipo de violência familiar ou do parceiro, em contraposição à Reátegui (2019), que apresentava uma incidência de cerca de 72\%. Tal dado pode ser analisado sob a perspectiva de que esse é um assunto delicado e que muitas vezes traz à tona insegurança e vergonha por parte da adolescente, podendo assim, apresentar um viés de pesquisa, ainda que os questionários sejam aplicados individualmente e as entrevistadas estejam respaldadas pelo sigilo da pesquisa.

Ainda em âmbito familiar, um ponto importante de análise é a educação sexual prestada pela família, ou até mesmo fora dela, nas escolas. Tema esse que ainda nos dias de hoje é tido como tabu para muitas famílias, o que dificulta o conhecimento dos adolescentes quanto aos métodos contraceptivos e demais informações (Munslinger, Silva, Bortoli, \& Guimarães, 2016).

Nesta pesquisa, cerca de $68,5 \%$ das entrevistadas afirmaram ter presenciado algum tipo de educação sexual na escola ou faculdade, dado esse que contrapõe a literatura, que mostra uma cerca dificuldade na transmissão de conhecimentos acerca da educação sexual para jovens adolescentes.

No tocante à unidade de saúde mais próxima, a distância e o tempo necessário para chegar até ela são importantes fatores para um acompanhamento pré-natal adequado. Esses são dados que inclusive devem ser identificados já na primeira consulta de pré-natal, de acordo com o Ministério da Saúde (2012) haja vista que uma dificuldade de acesso pode levar a gestante a realizar um acompanhamento inadequado, podendo acarretar em problemas futuros na gestação e parto. No presente artigo, apenas 5,6\% das gestantes afirmaram morar a mais de uma hora da unidade de saúde mais próxima, o que conflita com os dados observados por Ayele, Gebregzabher, Hailu, e Assefa (2018) que demonstravam que a maioria das gestantes demorava mais de uma hora para chegarem à uma unidade.

Em relação aos hábitos de vida, observou-se que, 66,6\% não faz uso de bebida alcoólica, e que, considerando a parcela das entrevistadas que faziam a ingesta do álcool antes da gestação, apenas 27,8\% dessa porcentagem continuou com o 
ato durante a gravidez. No que corresponde ao uso de cigarros, concluiu-se que apenas uma minoria $(7,4 \%)$ possui o costume de fumar, porém, dessa minoria, $75 \%$ continuaram fumando durante a gestação. Além disso, no que tange o consumo de drogas ilícitas durante a gestação, constatou-se uma porcentagem bem mínima de 1,9\%. Segundo dados obtidos por Moraes e Assunção (2020), mais de 70\% das gestantes adolescentes entrevistadas negaram o uso de álcool e cigarro durante a gestação, no entanto, nas participantes que afirmaram o consumo, o álcool e o tabaco ainda foram sobressalentes em relação às demais drogas, corroborando diretamente com os dados de nossa pesquisa.

Foi identificada também na amostra de Souza, Santos, Oliveira e Santos (2020) que as primeiras experiências às drogas das gestantes ocorreram em 61,5\% na faixa etária dos 10 aos 15 anos, sendo o álcool e o tabaco as principais drogas lícitas consumidas. No entanto, de acordo com o estudo de Usynina, Postoev, Odland, e Grjibovski (2018) tanto a ingestão de drogas lícitas quanto ilícitas possuíam maior recorrência em gestantes adolescentes, tal dado contradiz com o resultado aqui observado, em que apenas uma pequena porcentagem se apresentava usuária de tais substâncias. Outro dado a ser ponderado foi relatado no estudo de Cândido et al. (2019) no qual, 54\% das gestantes adolescentes consumiam bebidas alcóolicas e 80\% delas referiam o consumo da droga em gestações anteriores, podendo então ser afirmado, que a vulnerabilidade para o consumo do álcool persiste durante as gestações, ou seja, há relação de reincidência de gravidez precoce em quem habitualmente faz utilização de tal droga.

Quanto à religião, a literatura disponível não mostrou associações significativas com a gestação em adolescentes. Contudo, Alrahmani et al. (2016) afirmou que um dos fatores predisponentes para a gestação na adolescência é a falta de religião. Tal fato diverge com nossos dados, em que $85,2 \%$ das entrevistadas são adeptas a alguma, e dentre elas, $74 \%$ se consideram praticantes da religião.

\section{Conclusão}

O estudo em questão foi capaz de demonstrar a complexidade por trás dos aspectos sociais e familiares das gestantes adolescentes atendidas na Santa Casa de Anápolis-GO, ademais evidenciou a importância desse assunto nas discussões de saúde pública. Conclui-se que dentre os antecedentes familiares é comum a gestação na adolescência nos antecedentes da mãe da puérpera, o apoio familiar e do pai da criança é presente, e elas afirmam possuir um bom convívio em casa, além de uma gravidez não planejada.

Nos aspectos sociais, menos de um terço das adolescentes sofreu ou presenciou violência familiar, a maioria presenciou educação sexual na escola, prevalência de acesso oportuno a unidade de saúde, baixa, porém presente a ingestão de bebida alcoólica, cigarro e drogas ilícitas durante a gestação.

Dessa forma nota-se que apesar de um perfil social e familiar aparentemente adequado essas adolescentes ainda assim engravidaram em hora não oportuna o que indica a necessidade de analisar esses dados com cautela e a partir deles propor algumas intervenções. São elas: melhorar a metodologia das aulas de educação sexual, melhorar a relação médico-adolescente nas unidades de saúde, implementar programas locais onde esse problema for mais prevalente, melhorar a detecção de violência familiar, incentivar a comunicação intrafamiliar, ampliar as perspectivas de futuro das adolescentes.

Adicionalmente, fazem-se necessárias a realização de outras pesquisas para melhor elucidar a influência familiar e social na ocorrência de uma gravidez não planejada na adolescência, que possam aprofundar na subjetividade das relações familiares.

\section{Referências}

Almeida, A. H. d. V. d., Gama, S. G. N. d., Costa, M. C. O., Carmo, C. N. d., Pacheco, V. E., Martinelli, K. G., \& Leal, M. d. C. (2020). Prematuridade e gravidez na adolescência no Brasil, 2011-2012. Cadernos de Saúde Pública, 36, e00145919. 10.1590/0102-311x00145919 
Alrahmani, L., Abdelsattar, Z. M., Adekola, H., Puscheck, E., Gonik, B., \& Awonuga, A. O. (2016). Risk-profiles and outcomes of multi-foetal pregnancies in adolescent mothers. Journal of Obstetrics and Gynaecology, 36(8), 1056-1060. 10.1080/01443615.2016.1196477

Aragão, F. d. A., Jr., \& Cavalcanti, M. d. A. S. Educação em saúde no planejamento familiar e aconselhamento gestacional. UNA-SUS, 2019. https://ares.unasus.gov.br/acervo/handle/ARES/13740

Ayele, B. G. k., Gebregzabher, T. G., Hailu, T. T., \& Assefa, B. A. (2018). Determinants of teenage pregnancy in Degua Tembien District, Tigray, Northern Ethiopia: A community-based case-control study. PLoS One, 13(7), e0200898.10.1371/journal.pone.0200898

Bennett, T., Skatrud, J. D., Guild, P., Loda, F., \& Klerman, L. V. (1997). Rural adolescent pregnancy: a view from the South. Family Planning Perspectives, 256-267. https://pubmed.ncbi.nlm.nih.gov/9429870/

Bouzas, I. C. da S., Cader, S. A., \& Leao, L. (2014). Gravidez na adolescência: uma revisao sistemática do impacto da idade materna nas complicaçoes clínicas, obstétricas e neonatais na primeira fase da adolescência. Adolescencia e Saude, 11(3), 7-21. http://adolescenciaesaude.com/detalhe_artigo.asp?i $\mathrm{d}=457$ \&idioma $=$ Portugues

Cândido, T. C. R., Ferreira, G. C., Moreira, D. da S., Sousa, B. d. O. P., Cordeiro, S. M., Alfredo, E. R., \& Felipe, A. O. B. (2019). O uso de bebida alcoólica entre gestantes adolescentes. SMAD Revista Eletrônica Saúde Mental Álcool e Drogas (Edição em Português), 15(4), 1-8. https://doi.org/10.11606/issn.18066976.smad.2019.151701

Cunnington, A. J. (2001). What's so bad about teenage pregnancy? BMJ Sexual \& Reproductive Health, 27(1), 36-41. Doi: 10.1783/147118901101194877.

Departamento Científico de Adolescência. (2018) Guia Prático de Atualização. Prevenção da gravidez na adolescência. Adolesc. Saude, 15 (1), 86-94. https://cdn.publisher.gn1.link/adolescenciaesaude.com/pdf/v15s1a11.pdf' 1

Estrela, C. (2018). Metodologia Científica: Ciência, Ensino, Pesquisa. Editora Artes Médicas.

Gama, S. G. N. d., Szwarcwald, C. L., \& Leal, M. d. C. (2002). Experiência de gravidez na adolescência, fatores associados e resultados perinatais entre puérperas de baixa renda. Cadernos de Saúde Pública, 18(1), 153-161. https://doi.org/10.1590/S0102-311X2002000100016.

Goodyear, R. K., Newcomb, M. D., \& Locke, T. F. (2002). Pregnant Latina teenagers: Psychosocial and developmental determinants of how they select and perceive the men who father their children. Journal of Counseling Psychology, 49(2), 187. Doi: https://doi.org/10.1037/0022-0167.49.2.187

Henshaw, S. K., \& Feivelson, D. J. (2000). Teenage abortion and pregnancy statistics by state, 1996. Family Planning Perspectives, 272-280. $10.1363 / 3227200$

Lawlor, D. A., \& Shaw, M. (2004). Teenage pregnancy rates: high compared with where and when? Journal of the Royal Society of Medicine, 97(3), 121-123. 10.1258/jrsm.97.3.121

Levin, J. (1987). Estatística Aplicada para Ciências Humanas. Harbra.

Melhado, A., Sant'Anna, M. J. C., Passarelli, M. L. B., Coates, V. (2009). Impacto da atenção integral à mãe adolescente como fator de proteção à reincidência. Monteiro, DLM; Trajano, AJB; Bastos, AC: Gravidez e Adolescência, 59-63. https://cdn.publisher.gn1.link/adoles cenciaesaude.com/ pdf/v5n2a 09.pdf

Ministério da Saúde (2012). Cadernos de Atenção Básica. Atenção ao pré-natal de baixo risco. Série A. Normas e Manuais Técnicos

Cadernos de Atenção Básica, n 32. Brasília. Resgatado em http://bvsms.saude.gov.br/bvs/publicacoes/cadernos_atencao_basica_32_prenatal.pdf Ministério da Saúde (2021). Banco de dados do Sistema Único de Saúde-DATASUS. Recuperado em 4 junho, 2021 de http://www.datasus.gov.br Moraes, M. H. dos S., \& Assunção, M. d. J. S. M. (2020). Perfil socioeconômico e autocuidado das gestantes adolescentes. Research, Society and Development, 9(5), e69953091-e69953091. 10.33448/rsd-v9i5.3091

Munslinger, I. M., da Silva, S. M., Bortoli, C. d. F. C. de, \& Guimarães, K. B. (2016). A maternidade na perspectiva de mães adolescentes. Revista Brasileira em Promoção da Saúde, 29(3), 357-363. https://doi.org/10.5020/18061230.2016.p357

Reátegui, D. O. A. (2019). Influencia de la violencia familiar en la evolución del embarazo en adolescentes atendidas en el Centro de Salud de Morales 2018. Repositório Facultad de Ciencias de la Salud. http://repositorio.unsm.edu.pe/handle/11458/3093

Ribeiro, K. C. S. (2011). Adolescência e Sexualidade: Vulnerabilidade ás DSTs, HIV/Aids e a gravidez em adolescentes paraibanos. Repositório Institucional da UFPB. https://repositorio.ufpb.br/jspui/handle/tede/6878?locale=pt_BR

Sabroza, A. R., Leal, M. d. C., Gama, S. G. N. d., \& Costa, J. V. d. (2004). Perfil sócio-demográfico e psicossocial de puérperas adolescentes do Município do Rio de Janeiro, Brasil-1999-2001. Cadernos de Saúde Pública, 20, S112-S120. https://doi.org/10.1590/S0102-311X2004000700012.

Sadovsky, A. D. I. de, Matijasevich, A., Santos, I. S., Barros, F. C., Miranda, A. E., \& Silveira, M. F. (2018). Socioeconomic inequality in preterm birth in four Brazilian birth cohort studies. Jornal de Pediatria (Versão em Português), 94(1), 15-22. https://doi.org/10.1016/j.jped.2017.02.003

Santos, C. C. dos, Cremonese, L., Wilhelm, L. A., Castiglioni, C. M., \& Ressel, L. B. (2014). Perfil social de adolescentes gestantes e abandono escolar. Adolescencia e Saude, 11(3), 71-76. http://adolescenciaesaude.com/detalhe_artigo.asp?id=453

Santos, R. de C. A. N., Silva, R. M. da, Queiroz, M. V. O., Jorge, H. M. F. \& Brilhante, A. V. M. (2018). Realidades e perspectivas de mães adolescentes acerca da primeira gravidez. Revista Brasileira de Enfermagem, 71(1). https://doi.org/10.1590/0034-7167-2016-0444

Savegnago, S. D. O., \& Arpini, D. M. (2016). A Abordagem do Tema Sexualidade no Contexto Familiar: o Ponto de Vista de Mães de Adolescentes. Psicol. cienc. prof, 36(1). http://dx.doi.org/10.1590/1982-3703001252014 
Research, Society and Development, v. 10, n. 6, e24910615633, 2021

(CC BY 4.0) | ISSN 2525-3409 | DOI: http://dx.doi.org/10.33448/rsd-v10i6.15633

Silva, B. R. da, Neto (2019). Ciências da Saúde: Da Teoria à Prática 8. Atena Editora. 10.22533/at.ed.009191306

Souza, V. de M., Santos, R. da S., Oliveira, E. B. de, \& Santos, I. M. M. dos (2020). Mães adolescentes em consumo de álcool e outras drogas: estudo descritivo. Research, Society and Development, 9(8), e774986359-e774986359. 10.33448/rsd-v9i8.6359

Usynina, A. A., Postoev, V., Odland, J. Ø., \& Grjibovski, A. M. (2018). Adverse pregnancy outcomes among adolescents in North west Russia: a population registry-based study. International journal of environmental research and public health, 15(2), 261. 10.3390/ijerph15020261 